\title{
Longitudinal Dynamic Network Analysis Using the Over Time Viewer Feature in ORA
}

\author{
Ian McCulloh \& Kathleen Carley
}

March 9, 2009

CMU-ISR-09-118

\author{
Institute for Software Research \\ School of Computer Science \\ Carnegie Mellon University \\ Pittsburgh, PA 15213
}

This work was supported, in part, by the Army Research Institute for the Behavioral and Social Sciences, Army Project No. 611102B74F, the Army Research Lab ADA CTA - DAAD19-01-2-0009, the National Science Foundation (NSF) Integrative Graduate Education and Research Traineeship (IGERT) program [IGERT - DGE-9972762], and the Office of Naval Research [ONR MURI - N000140811186; ONR MMV - N00014-06-1-0104; ONR SORASCS - N000140811223]. This work is part of the Dynamics Networks project at the center for Computational Analysis of Social and Organizational Systems (CASOS) http://www.casos.cs.cmu.edu) in the School of Computer Science (SCS) at Carnegie Mellon University (CMU). The views and conclusions contained in this document are those of the authors and should not be interpreted as representing the official policies, either expressed or implied, of the Army Research Institute, the Army Research Lab, the National Science Foundation, the Office of Naval Research or the U.S.

government 
Keywords: networks, change detection, network evolution, longitudinal network analysis, dynamic network analysis. 


\begin{abstract}
Analyzing network over time has become increasingly popular as longitudinal network data becomes more available. Longitudinal networks are studied by sociologists to understand network evolution, belief formation, friendship formation, diffusion of innovations, the spread of deviant behavior and more. Organizations are interested in studying longitudinal network in order to get inside the decision cycle of major events. Prior to important events occurring in an organization, there is likely to exist an earlier change in network dynamics. Being able to identify that a change in network dynamics has occurred can enable managers to respond to the change in network behavior prior to the event occurring and shape a favorable outcome.

The Over Time Viewer is a software tool hosted by the CASOS software suite that enables the analysis of longitudinal dynamic network data. This report introduces the Over Time Viewer and provides instruction on how to effectively use its features. We provide step-by-step instructions and illustrations as well as a description of the technology underlying the tool.
\end{abstract}




\section{Table of Contents}

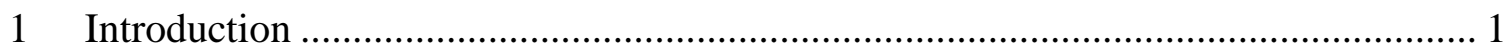

1.1 Importance of Change in Longitudinal Social Networks...................................... 2

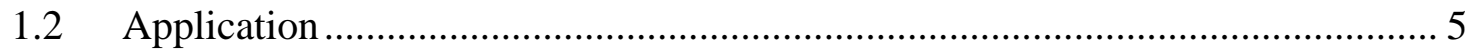

2 Using the Over Time Viewer................................................................................ 7

2.1 STEP 1: Launching the Over Time Viewer ………………................................. 7

2.2 STEP 2: Over Time Dependence .............................................................. 9

2.3 STEP 3: Network Change Detection.............................................................. 15

3 Future Work .................................................................................................... 16

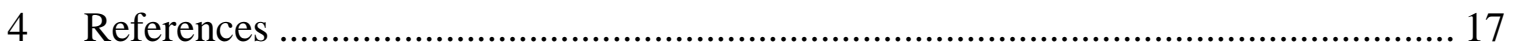




\section{Introduction}

Terrorists from al-Qaeda attacked America on 11 September 2001. Some suggest that these terrorists began to plan and resource this attack as early as 1997. If social network analysts could monitor the social, email, or phone networks of these terrorists and detect organizational changes quickly, they may enable military leaders to respond prior to the successful completion of their attack. Social network change detection (SNCD) is a novel approach to this problem. It combines the area of statistical process control and social network analysis. The combination of these two disciplines is likely to produce significant insight into organizational behavior and social dynamics.

Statistical process control is a statistical approach for detecting anomalies in the behavior of a stochastic process over time. This approach is widely used in manufacturing as a means for quality control. Manufacturing systems experience similar issues of high correlation, dependence, and non-ergodicity that is common in relational network data. I posit that applying statistical process control to graph-level network measures is effective at rapidly detecting changes in longitudinal network data.

It is important to note that I am not predicting change, but rather detecting that a change occurred quickly and making some inference about the actual time of change. For example, before a terrorist commits an attack, there will be a change in the social network as the organization plans and resources the attack. SNCD may allow an analyst to detect the change in the social network, prior to the successful completion of the attack. In a similar fashion, corporate managers may wish to detect changes in the organizational behavior of their companies to capitalize on innovation or prevent problems. For example, the CEO of Dupont became aware of the U.S. recession in late 2008 in time to enact a crisis management plan averting financial disaster for the company. In this example, the economic change had already occurred. Dupont's success was not in predicting a recession, but rather detecting that it had occurred quickly, in time to respond.

SNCD may offer executives and military analysts a tool to operate inside the normal decision cycle. Figure 1 represents some measure of interest over time. It could be the revenue of a company, the combat power of an enemy, or for our purposes a measure of interest from a social network. When do we conclude from this measure that a change may have occurred? Let us assume that by conventional methods we can detect a change in organizational behavior as of "today", the vertical line in Figure 1. This time point might be too late to take and preventative or mitigating action. In other words, this could be the point of inevitable bankruptcy for the company, or the successful culmination of a terrorist attack. Identifying that a change occurred by time period E might allow the analyst to respond to the change before it is too late; get inside the decision cycle.

Change detection is more challenging than it may seem at first. We can see a sudden change in the measure between time $\mathrm{D}$ and time $\mathrm{E}$, however, this may look very similar to the peak at time A. Furthermore, if we assert that a change in fact occurs at time A, 
there may exist a large amount of time periods to investigate for the cause of any change. If we can identify more likely points in time where change may have occurred, we can reduce the costs in terms of time and resources to search for the potential causes of change. Identifying the likely time that a change may have occurred is called change point identification.

Another problem that we face is detecting the change as quickly as possible after the change occurred. Can we improve the ability to get inside the decision cycle by detecting the change at time $\mathrm{D}$, or even better at time $\mathrm{B}$ ? This is called change detection. This thesis is a first attempt to investigate this challenging problem in longitudinal network analysis.

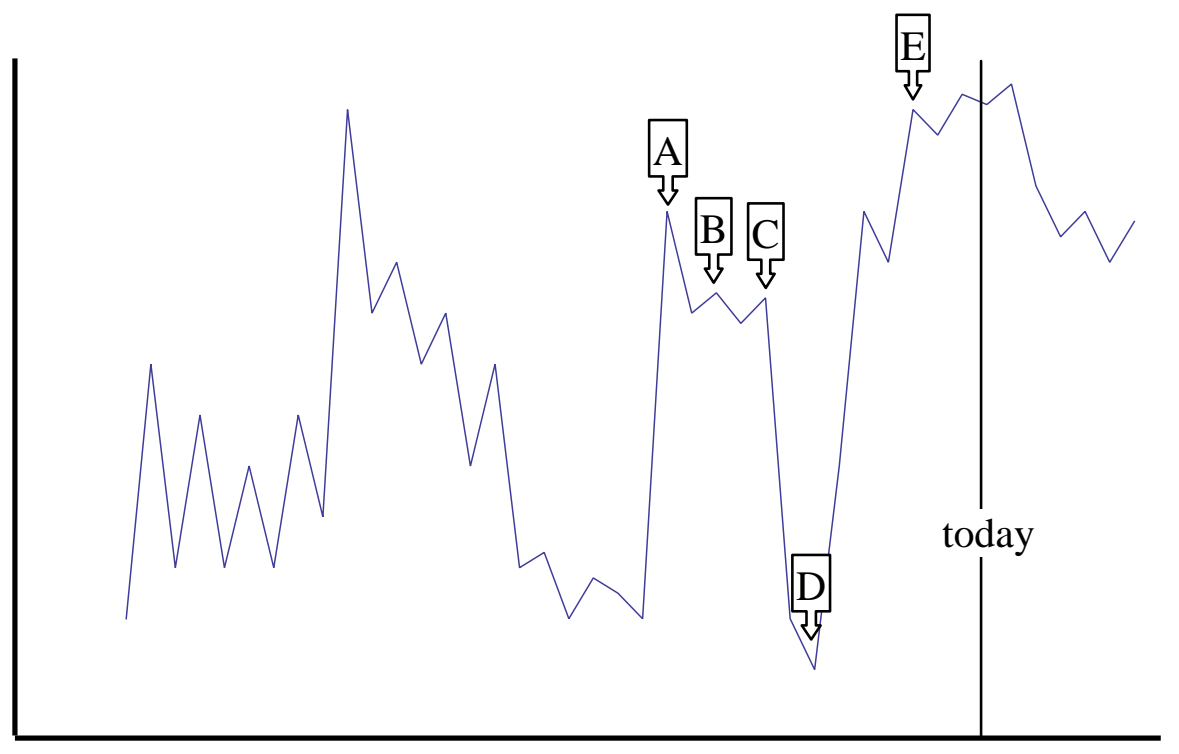

Figure 1. Example of Change Detection

\subsection{Importance of Change in Longitudinal Social Networks}

This thesis addresses a new area of research that is a national need. Research agencies throughout the Department of Defense (DoD) and the U.S. Government have demonstrated recent interest in pursuing research in the area of social network analysis. Particular interest is in stochastic and predictive modeling of these networks. The National Research Council (NRC) (2005) in a recent report on Network Science identified a lack of understanding in the stochastic behavior of networks. They further stated that there existed a great need for this understanding in order to develop effective predictive models. Twenty percent of the research tasks in the Office of Naval Research's (ONR) recent broad agency announcement 07-036 were in the area of social networks. One of the research tasks were for "real time methods for the analysis of networks." Another task was to develop "metrics extracted in real time to diagnose effective or ineffective collaboration or negotiation," and for creating "unobtrusive data collection methodologies" for social networks. The U.S. Army Research Institute for the 
Behavioral and Social Sciences (ARI) has requested research in social networks to "investigate individual unit and organizational behavior within the context of complex networked environments” in their fiscal year 2008 BAA. The U.S. Army Research Office has already budgeted over \$1 Million per year for faculty and cadets at the U.S. Military Academy to study the stochastic behavior of networks. The National Academies identified the need for research in this area as early as 2003 in the Dynamic Social Network Modeling and Analysis workshop in Washington, DC.

While this research will not predict network behavior, it will provide an approach for more accurately detecting that a change occurred and when that change likely occurred. This is an important first step for any predictive analysis. If a social scientist can accurately detect change and the time change occurred, only then can he investigate the cause of change with any real success. Therefore, I posit that this approach will contribute to longitudinal network analysis in general, enabling future researchers to address the problem of prediction.

Much research has been focused in the area of longitudinal social networks (Sampson, 1969; Newcomb, 1961; Sanil, Banks, and Carley, 1995; Snijders, 1990, 2007; Frank, 1991; Huisman and Snijders, 2003; Johnson et al, 2003; McCulloh et al, 2007a, 2007b). Wasserman et al. (2007) state that, "The analysis of social networks over time has long been recognized as something of a Holy Grail for network researchers." Doreian and Stokman (1997) produced a seminal text on the evolution of social networks. In their book they identified as a minimum, 47 articles published in Social Networks that included some use of time, as of 1994. They also noted several articles that used over time data, but discarded the temporal component, presumably because the authors lacked the methods to properly analyze such data. An excellent example of this is the Newcomb (1961) fraternity data, which has been widely used throughout the social network literature. More recently, this data has been analyzed with its' temporal component (Doreian et al., 1997; Krackhardt, 1998).

Methods for the analysis of over time network data has actually been present in the social sciences literature for quite some time (Katz and Proctor, 1959; Holland and Leinhardt, 1977; Wasserman, 1977; Wasserman and Iacobuccci 1988; Frank, 1991). The dominant methods of longitudinal social network analysis include Markov chain models, multi-agent simulation models, and statistical models. Continuous time Markov chains for modeling longitudinal networks were proposed as early as 1977 by Holland and Leinhardt and by Wasserman. Their early work has been significantly improved upon (Wasserman, 1979; 1980; Leenders, 1995; Snijders and van Duijn, 1997; Snijders, 2001; Robins and Pattison, 2001) and Markovian methods of longitudinal analysis have even been automated in a popular social network analysis software package SIENA. A related body of research focuses on the evolution of social networks (Dorien, 1983; Carley, 1991; Carley, 1995; Dorien and Stokman, 1997) to include three special issues in the Journal of Mathematical Sociology (JMS Vol 21, 1-2; JMS Vol 25, 1; JMS Vol 27, 1). Evolutionary models often use multi-agent simulation. Others have focused on statistical models of network change (Feld, 1997; Sanil, Banks, and Carley, 1995; Snijders, 1990, 1996; Van de Bunt et al, 1999; Snijders and Van Duijn, 1997). Robins and Pattison 
(2001, 2007) have used dependence graphs to account for dependence in over-time network evolution. We can clearly see that the development of longitudinal network analysis methods is a well established problem in the field of social networks. Table 1 provides a comparison of the dominant methods for longitudinal network analysis.

The literature shows that there exist four network dynamic states in longitudinal social networks. A network can exhibit stability. This occurs when the underlying relationships in a group remain the same over time. Observations of the network can vary between time periods due to observation error, survey error, or normal fluctuations in communication. A network can evolve. This occurs when interactions between agents in the network cause the relationships to change over time. A network can experience shock. This type of change is exogenous to the social group. Finally, a network can experience a mutation. This occurs when an exogenous change initiates evolutionary behavior.

Much of the research in longitudinal social networks has focused on evolutionary change. Markov methods and multi-agent simulation are effective at helping social scientists understand evolutionary change. However, a careful review of the literature did not reveal any research in detecting shock or mutations in the network.

SNCD provides a statistical approach for detecting changes in a network over time. In addition to change detection, change point identification is also possible. Identifying changes and change points in empirical data, will allow social scientists to better isolate factors affecting network evolution as well as the relatively new concept of shock. Moreover, knowing when a network change occurs provides an analyst insight in how to bifurcate longitudinal network data for analysis.

A complete review of methods for longitudinal social network analysis is beyond the scope of this thesis. The reader is referred to Wasserman and Faust (1994); Dorien and Stokman (1997); and Carrington, Scott and Wasserman (2007). Essentially, methods for longitudinal social network analysis have been focused on modeling and testing for the significance of social theories in empirical data. These methods have not been designed to detect change over time. This thesis is focused on detecting change in a social network over time. 


\begin{tabular}{|c|c|c|c|c|}
\hline & Markov Chain & Multi-Agent & Statistical & SNCD \\
\hline $\begin{array}{l}\text { Problem } \\
\text { Addressed }\end{array}$ & $\begin{array}{l}\text { 1. Network } \\
\text { evolution based } \\
\text { on Markovian } \\
\text { assumptions. } \\
\text { 2. Determine } \\
\text { how underlying } \\
\text { social theories } \\
\text { affect group } \\
\text { dynamics. }\end{array}$ & $\begin{array}{l}\text { 1. Network } \\
\text { evolution based } \\
\text { on node-level } \\
\text { behavior. } \\
\text { 2. Evaluate the } \\
\text { impact of social } \\
\text { intervention on } \\
\text { group } \\
\text { dynamics. }\end{array}$ & $\begin{array}{l}\text { 1. Compare the } \\
\text { properties of } \\
\text { networks at } \\
\text { different points } \\
\text { in time. }\end{array}$ & $\begin{array}{l}\text { 1. Detect } \\
\text { change (shock, } \\
\text { evolution, or } \\
\text { mutation) over } \\
\text { time in } \\
\text { empirical } \\
\text { networks. }\end{array}$ \\
\hline $\begin{array}{l}\text { Key } \\
\text { Assumptions }\end{array}$ & $\begin{array}{l}\text { 1. Future } \\
\text { behavior of } \\
\text { network is } \\
\text { independent of } \\
\text { the past. } \\
2 \text {. There is no } \\
\text { exogenous } \\
\text { change in the } \\
\text { network. }\end{array}$ & $\begin{array}{l}\text { 1. Node level } \\
\text { behavior can } \\
\text { drive group } \\
\text { behavior. } \\
\text { 2. Underlying } \\
\text { social theories } \\
\text { affecting group } \\
\text { dynamics are } \\
\text { known. }\end{array}$ & $\begin{array}{l}\text { Assumptions } \\
\text { vary, but } \\
\text { include such } \\
\text { things as dyadic } \\
\text { independence/ } \\
\text { dependence, } \\
\text { over-time } \\
\text { independence, } \\
\text { one node class. }\end{array}$ & $\begin{array}{l}\text { Group behavior } \\
\text { can be inferred } \\
\text { from } \\
\text { longitudinal } \\
\text { social networks }\end{array}$ \\
\hline $\begin{array}{l}\text { Limitations for } \\
\text { change detection }\end{array}$ & $\begin{array}{l}\text { 1. Does not } \\
\text { account for } \\
\text { exogenous } \\
\text { change. } \\
\text { 2. Markov } \\
\text { assumption. }\end{array}$ & $\begin{array}{l}\text { 1. Used to } \\
\text { model both } \\
\text { exogenous and } \\
\text { evolutionary } \\
\text { change, but not } \\
\text { to detect } \\
\text { change. } \\
2 \text {. Underlying } \\
\text { social theories } \\
\text { must be known. }\end{array}$ & $\begin{array}{l}\text { 1. Does not } \\
\text { handle over- } \\
\text { time } \\
\text { dependence. } \\
\text { 2. Not a } \\
\text { longitudinal } \\
\text { approach. }\end{array}$ & $\begin{array}{l}\text { 1. Ergodicity } \\
\text { and dependence } \\
\text { is not fully } \\
\text { addressed. }\end{array}$ \\
\hline Strengths & $\begin{array}{l}\text { Determining } \\
\text { significant } \\
\text { social theories } \\
\text { affecting group } \\
\text { dynamics. }\end{array}$ & $\begin{array}{l}\text { Simulating } \\
\text { group dynamics } \\
\text { in a social } \\
\text { network. }\end{array}$ & $\begin{array}{l}\text { Comparing } \\
\text { social } \\
\text { networks. }\end{array}$ & $\begin{array}{l}\text { Detecting } \\
\text { changes in } \\
\text { empirical social } \\
\text { networks over } \\
\text { time. }\end{array}$ \\
\hline
\end{tabular}

\subsection{Application}

This thesis will provide insight into the stochastic behavior of social networks. In addition, algorithms will be proposed that detect subtle changes in a social network. Imagine Joe Analyst working in an intelligence center trying to understand the dynamics of global terrorism. He currently has wide array of tools to assist him. He can piece together social networks from news papers and broadcasts, intercepted voice communication, and intelligence gathered from field agents. He can model this 
information with social networks and use various measures to identify individuals who are well connected, influential, or connect otherwise disconnect terrorist cells. In other words, he can tell you who was likely responsible for an attack in the past, and who was influential in the organization. But, what about today? Have influential members become less important? Are other members of the organization assuming more influential positions in the social network? Can we detect a change in the social network of a terrorist organization as they increase their communication before they are able to execute their planned terrorist attack? These are the questions that this research will help answer.

Applications are not limited to the military. Consider a civilian company, whose managers can identify major leadership challenges before they affect the productivity of the company. The introduction of e-mail and cell phones into the workplace has significantly changes the dynamics of communication. In the past, workers had limited peers available that they could ask about problems, before they had to seek guidance from senior management. Today, the available peers to consult are limited only by a person's social network. With growing on-line communities of practice, this network is becoming larger and larger. While this is good that workers are able to resolve problems at a lower level, senior managers are unable to influence decisions with their senior judgment and experience. This research will provide those managers with a tool to detect potential problems in their organization, by detecting subtle changes in the social network of employees. 


\section{Using the Over Time Viewer}

This section provides step-by-step instructions on how to use the Over Time Viewer in ORA to conduct longitudinal network analysis. This procedure is illustrated with a longitudinal network data set constructed from email activity. The data set is part of McCulloh's IkeNet 3, (McCulloh, 2009). The IkeNet data set consists of longitudinal network data constructed from email traffic on a group of officers and cadets at the U.S. Military Academy. The participants agreed to allow the researchers to monitor their email activity in exchange for the use of a blackberry. Daily networks were created, where the nodes were the participants and their email messages. There were directed links from a node sending an email to the email message that they sent and from the email message to the recipients of the message, creating a bipartite network. For this report, we conducted a relational algebra in ORA to multiply the (agent x email) network by the (email x agent) network to create a social network, where individuals were related with a weight corresponding to the number of email messages sent between individuals.

\subsection{STEP 1: Launching the Over Time Viewer}

To analyze networks over time, several features have been created in ORA. Before attempting to use the Over Time Viewer, the analyst must first load the metanetworks corresponding to different time periods into ORA. The meta-networks can be time stamped in ORA, otherwise, they must be loaded in the order of the correct time sequencing. Once the networks are loaded into ORA, the longitudinal analysis features can be found in the Over-Time Viewer which can be accessed from the pull down menu.

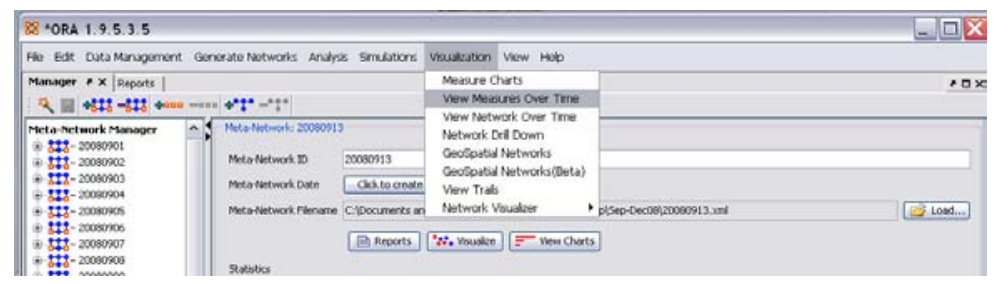

A pop-up window will appear, asking the user how to conform the networks. When you have multiple networks over time, some nodes may appear in certain time periods and be absent from others. The user must therefore decide whether to:

a) include nodes as isolates in time periods where they are not observed (union);

b) exclude nodes that are not in all time periods (intersection);

c) calculate graph level measures on the networks as they are (do nothing).

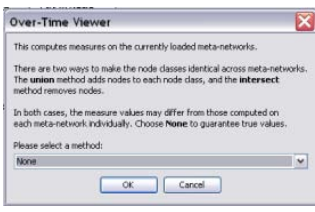


ORA will spend a bit of time calculating measures and then the following screen will appear.

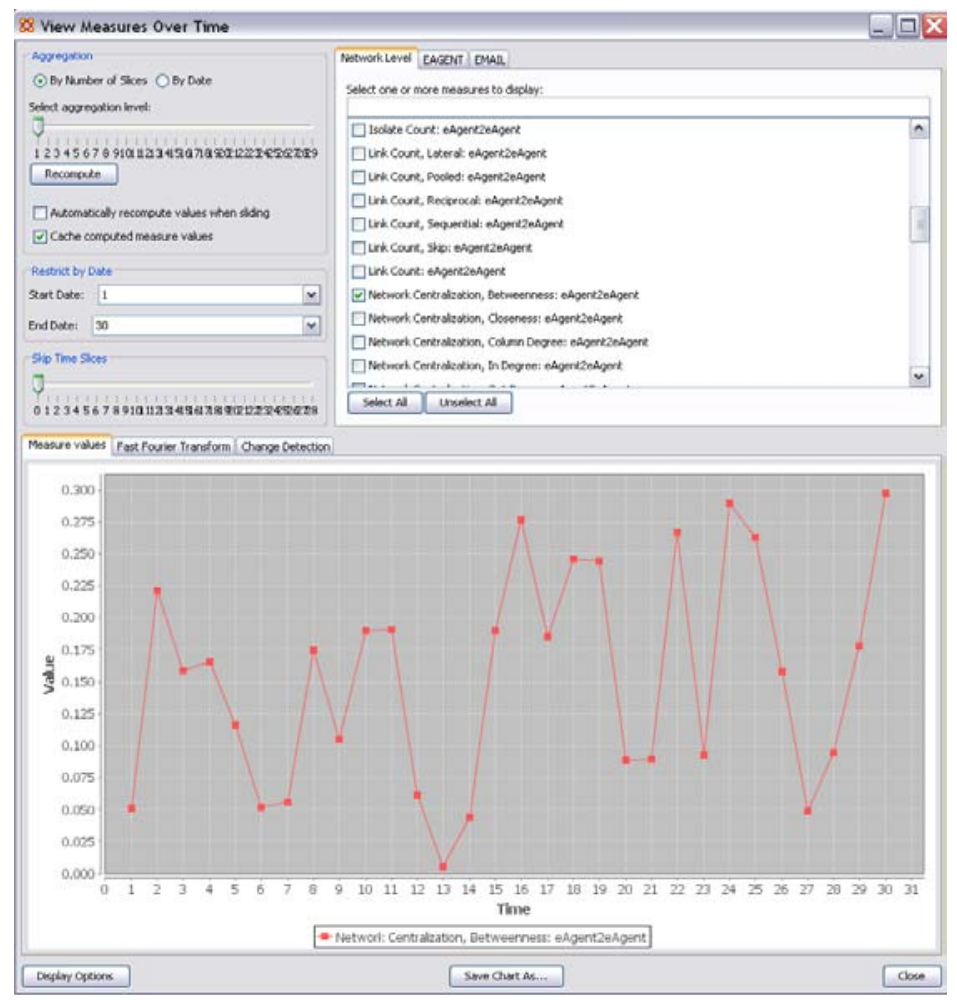

The data used in this example comes from IkeNet 3. The networks analyzed are daily snapshots of the network.

In the upper left the user can make some choices about the aggregation level. In other words, if you have daily networks, do you want to aggregate over 7 time periods to have weekly networks? Or perhaps you would prefer every 3 days.

Below this are some options to restrict the time periods that the analyst wants to look at. This is particularly useful when there are many more than about 30 time periods. This can also be used, even if the dates are not recorded for the networks. If no dates are recorded, integer time periods are assigned to the networks beginning with time 1 . An example of networks with no dates recorded is shown in the screen capture above.

In the upper right of the Over Time Viewer, the analyst will choose the particular measures that they want to analyze. So far, we have only explored network level measures. Theoretically, there is no reason why this will not work for any node level or meta network measure. Therefore, this capability is included in ORA. We provide this disclaimer that analysis of agent level measures has not been proven as of the date of this publication. 


\subsection{STEP 2: Over Time Dependence}

One major obstacle to the study of network dynamics is periodicity or over-time dependence in longitudinal network data. For example, if we define a social network link as an agent sending an email to another, we have continuous time stamped data. Intuitively, we can imagine that individuals are more likely to email each other at certain times of the day, days of the week, etc. If the individuals in the network are students, then their email traffic might follow the school's academic calendar. Seasonal trends in data are common in a variety of other applications as well. When these periodic changes occur in the relationships that define social network links, social network change detection methods are more likely to signal a false positive. A false positive occurs when the social network change detection method indicates that a change in the network may have occurred, when in fact there has been no change. To illustrate, assume that we are monitoring the density of the network for change in hourly intervals. The density of the network measured for the interval between 3 A.M. and 4 A.M. might be significantly less than the network measured from 3 P.M. to 4 P.M. because most of the people in the network are asleep and not communicating between 3 A.M. and 4 A.M. This behavior is to be expected, however, and it is not desireable for the change detection algorithm to signal a potential change at this point. Rather, it would be ideal to control for this phenomenon by accounting for the time periodicity in the density measure. Only then can real change be identified quickly in a background of noise.

Periodicity can occur in many kinds of longitudinal data. Organizations may experience periodicity as a result of scheduled events, such as a weekly meeting or monthly social event. Social networks collected on college students are likely to have periodicity driven by both the semester schedule and academic year. Even the weather may introduce periodicity in social network data, as people are more or less likely to email, or interact face-to-face. At the U.S. Military Academy, people tend to run outside in warm weather in small groups of two or three. During the winter, people go to the gym, where they are likely to see many people. This causes an increase in face-to-face interaction as people stay inside. In a similar fashion, during the Spring and Fall, many people participate in inter-unit sporting events such as soccer, or Frisbee football. This can also affect people' face-to-face interaction and thus the social network data collected on them.

Spectral analysis provides a framework to understand periodicity. Spectral analysis is mathematical tool used to analyze functions or signals in the frequency domain as opposed to the time domain. If we look at some measure of a social group over time, we are conducting analysis in the time domain. The frequency domain allows us to investigate how much of the given measure lies within each frequency band over a range of frequencies. For example, Figure 11 shows a notional measure on some madeup group in the time domain. It can be seen that the measure is larger at points $B$ and $D$ corresponding to the middle of the week. The measure is smaller at points $\mathrm{A}, \mathrm{C}$, and $\mathrm{E}$. 


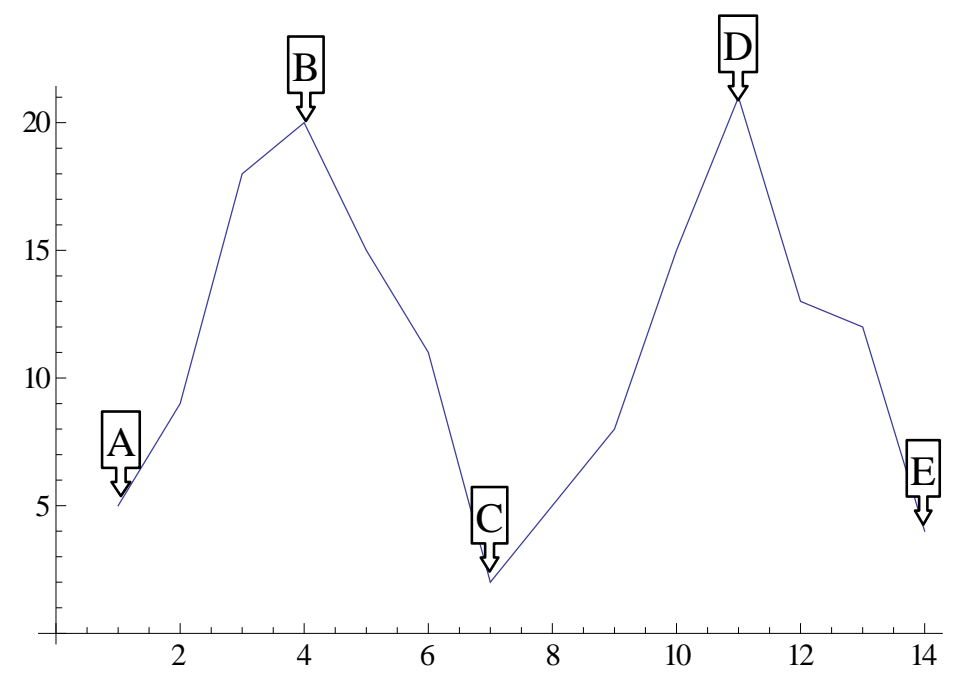

Figure 2 Notional Measure in Time Domain

If the signal in Figure 11 is converted to the frequency domain as shown in Figure 12, we can see how much of the measure lies within certain frequency bands. The negative spike in Figure 12 corresponds to 7 days, which is the weekly periodicity in the notional signal. The actual frequency signal only runs to a value of 8 on the $\mathrm{x}$-axis in Figure 12. The frequency domain signal after a value of 8 is a mirror image, or harmonic of the actual frequency signal.

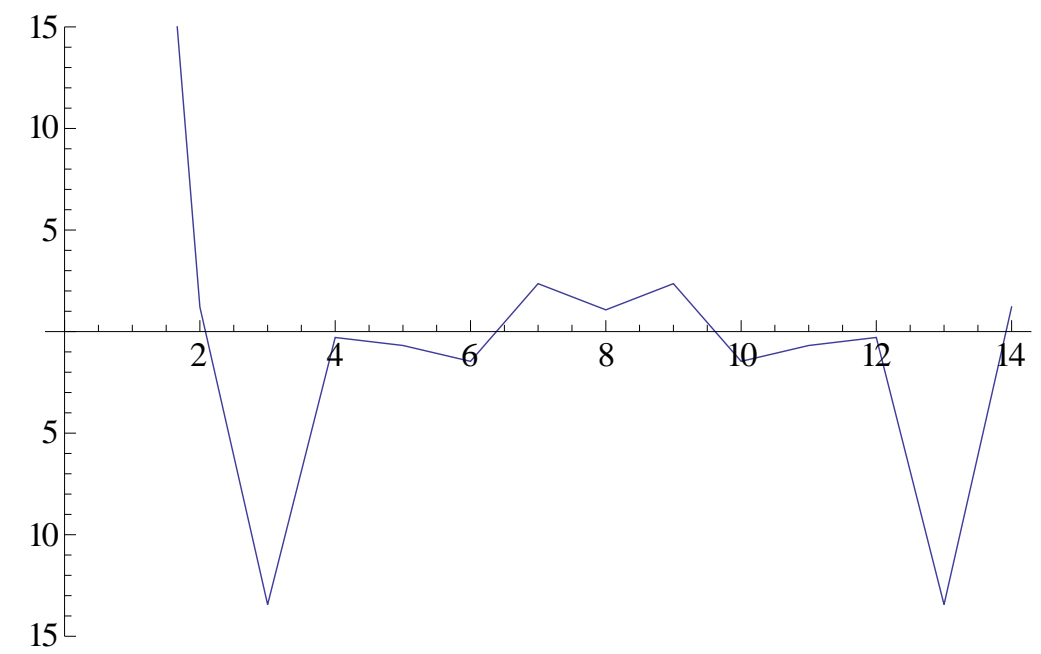

Figure 3. Notional Measure in Frequency Domain

The frequency domain representation of a signal also includes the phase shift that must be applied to a summation of sine functions to reconstruct the original over-time signal. In other words, we can combine daily, weekly, monthly, semester, and annual periodicity to recover the expected signal over-time due to periodicity. For example, Figures 13-15 represent monthly, weekly, and sub-weekly periodicities. If these signals are added together, meaning that the observed social network exhibits all three of these periodic behaviors, the resulting signal is shown in Figure 16. 


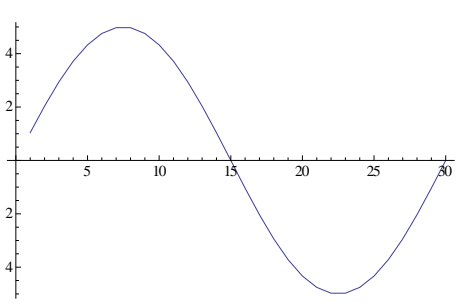

Figure 4. Monthly Period

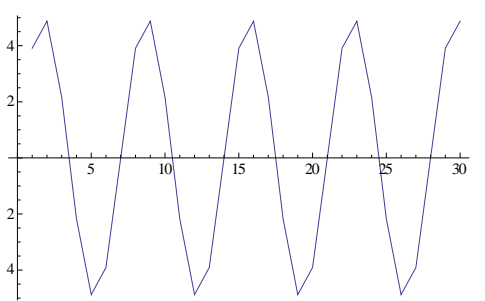

Figure 5. Weekly Period

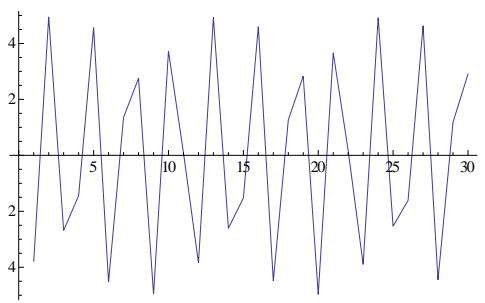

Figure 6. Sub-weekly Period

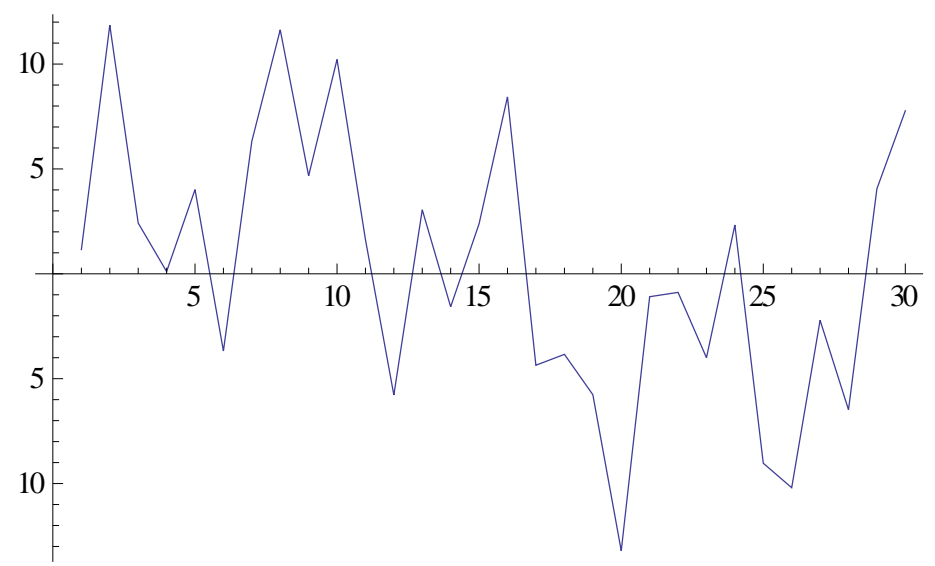

Figure 7. Sum of the Signal in Figures 13-15

If the periodicity in the signal shown in Figure 16 is not accounted for, it appears that there may be a change in behavior around time period 20, where the signal is negatively spiked. In reality, this behavior is caused by periodicity. If we transform the signal to the frequency domain as shown in Figure 17, we can see the weekly periodicity at point $\mathrm{B}$ and the sub-weekly periodicity at point $\mathrm{A}$.

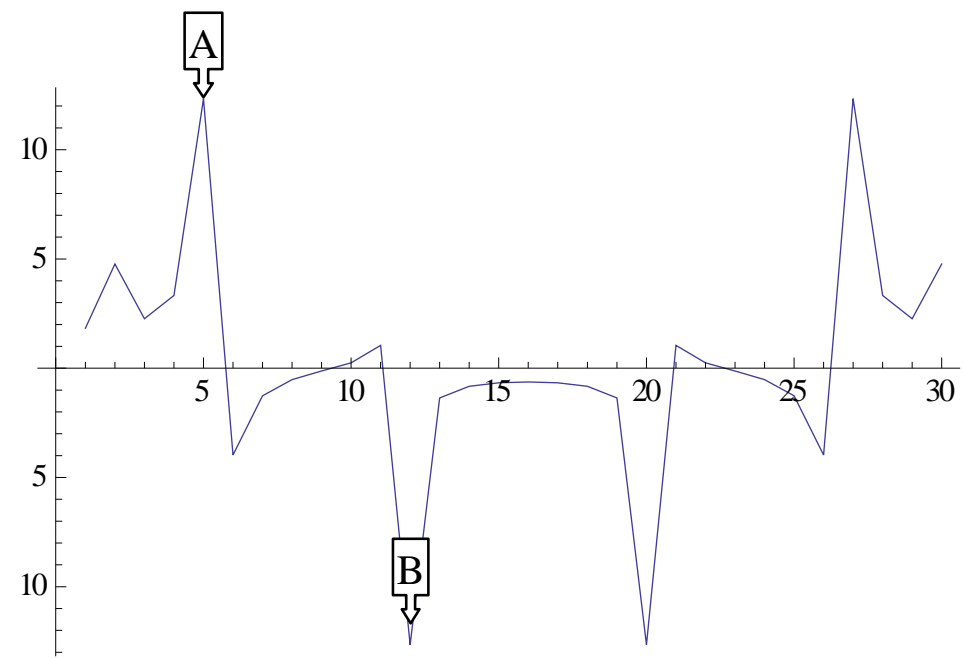

Figure 8. Transformation of Figure 16 to the Frequency Domain 
I propose that spectral analysis applied to social network measures over time will identify periodicity in the network. I will transform an over time network measure from the time domain to the frequency domain using a Fourier transform. I will then identify significant periodicity in the over-time network and present two methods for handling the periodicity.

The over time dependence analysis is accessed by selecting the Fast Fourier Transform tab in the Over Time Viewer as shown below. This displays the frequency plot of the data.

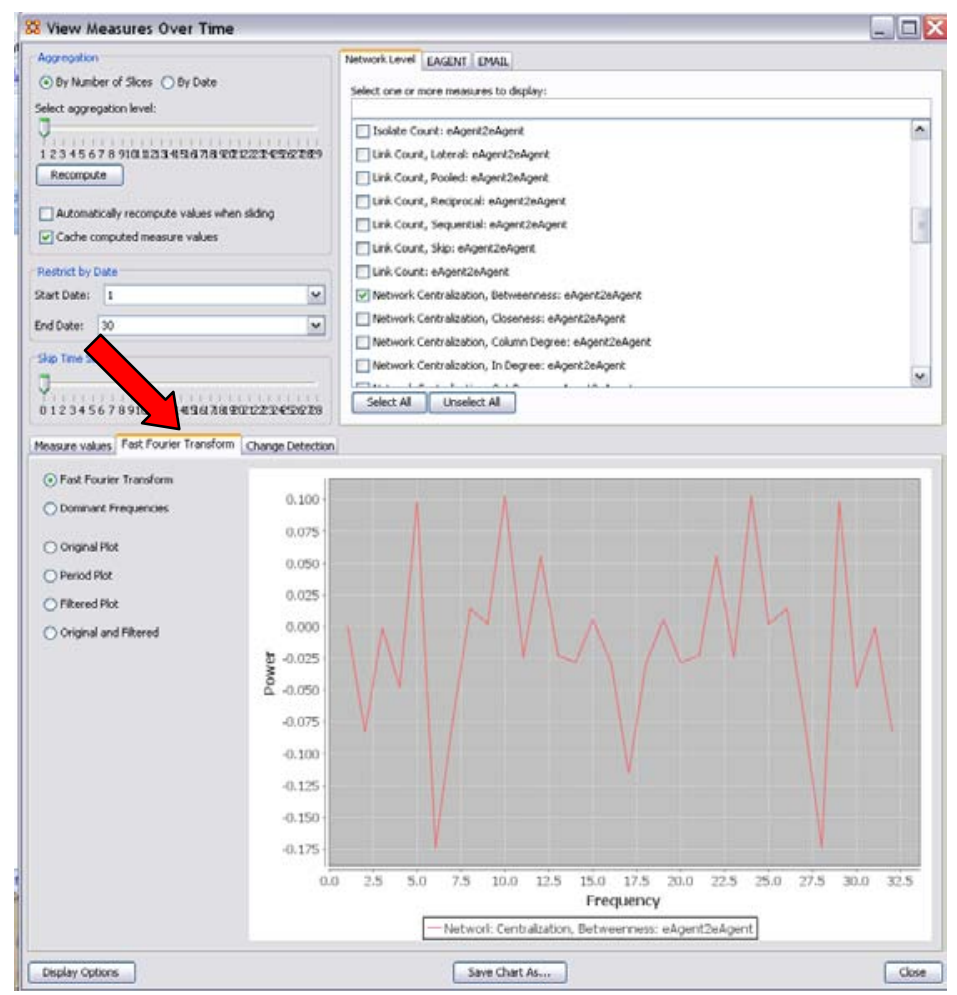

The analyst can use the Over Time Viewer to help determine which frequencies are significant. Selecting Dominant Frequencies on the radio button to the lower left, displays only the statistically significant frequencies. The Fourier Transform uses the normal distribution in order to transform data from the time domain to the frequency domain. Therefore, the normal distribution is an appropriate distribution to fit to the frequencies plotted in the frequency plot. All frequencies that are within two standard deviations of the mean are then set equal to zero for the dominant frequency plot, revealing only the dominant frequencies. A dominant frequency is a potential source of periodicity, as opposed to random noise in the over time signal. 


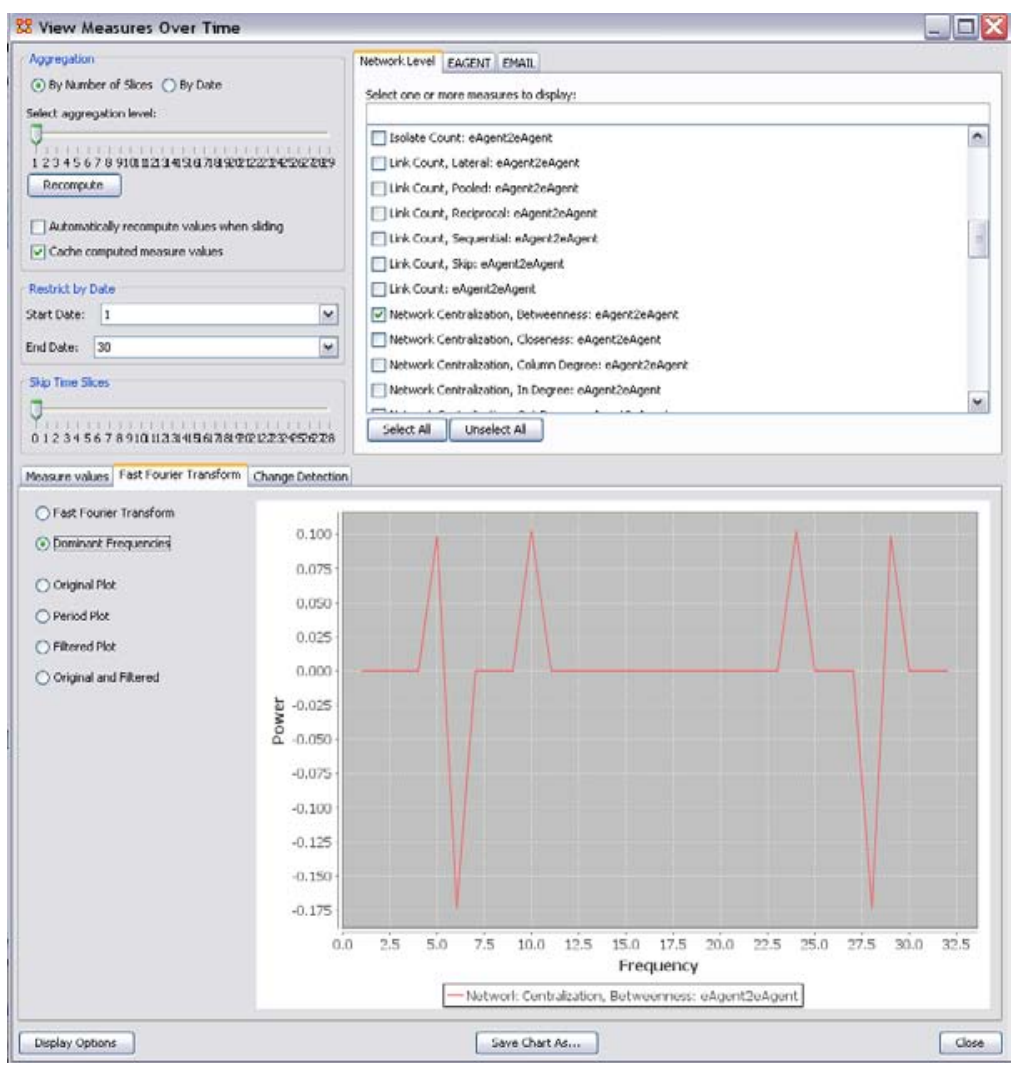

The analyst will often want to transform the statistically significant frequencies from the frequency domain back into the time domain so that he/she can make better sense of them. To do this, the analyst must select the radio button on the lower left called Period Plot.

The period plot shows the analyst the expected periodicity in the over-time data. In the example, you can see weekly periodicity. The peaks and valleys in the period plot occur approximately every 7 days. At this point, the analyst may wish to merge the daily data into weekly networks. This would average out the effects of weekends and evenings that are likely to affect the properties of daily networks. Another approach is to simply look at the networks departure from what is expected. 


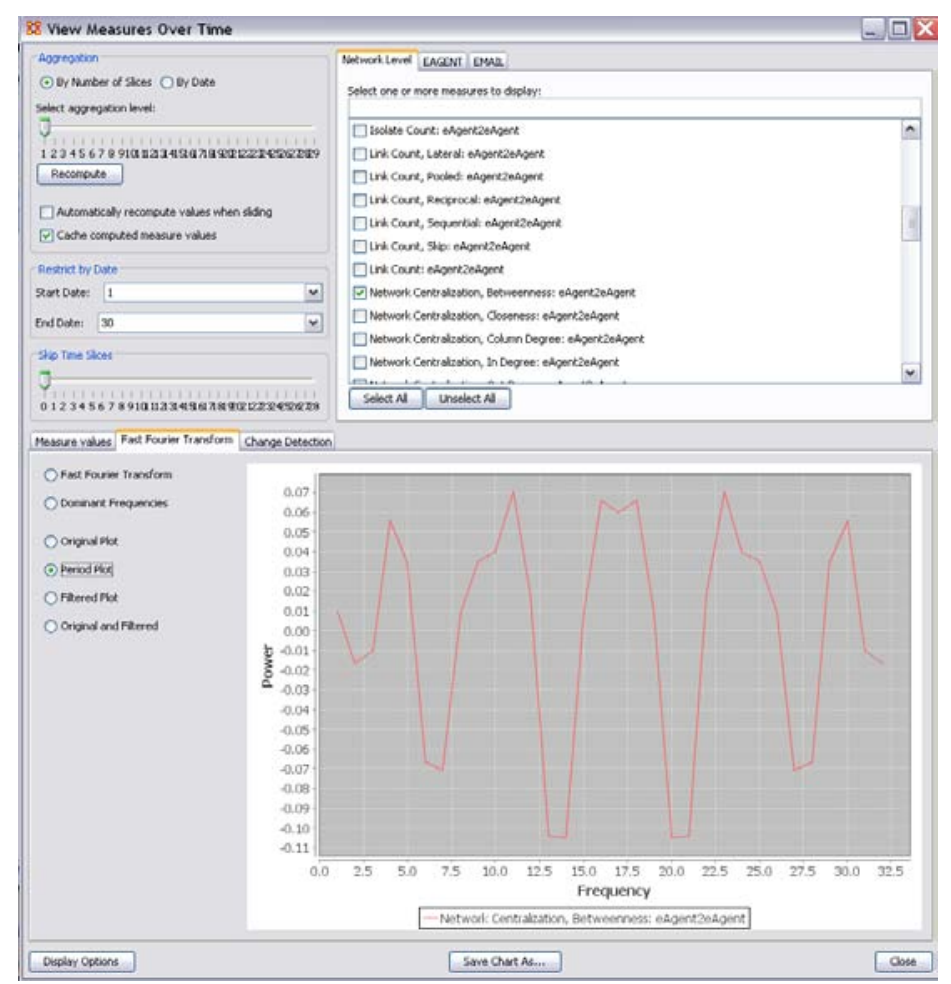

The Filtered Plot radio button will create an over-time plot of how the measure deviates from what is expected, based on the periodicity of the measure. You can also plot the filtered measure with the original measure to see the difference as shown below.

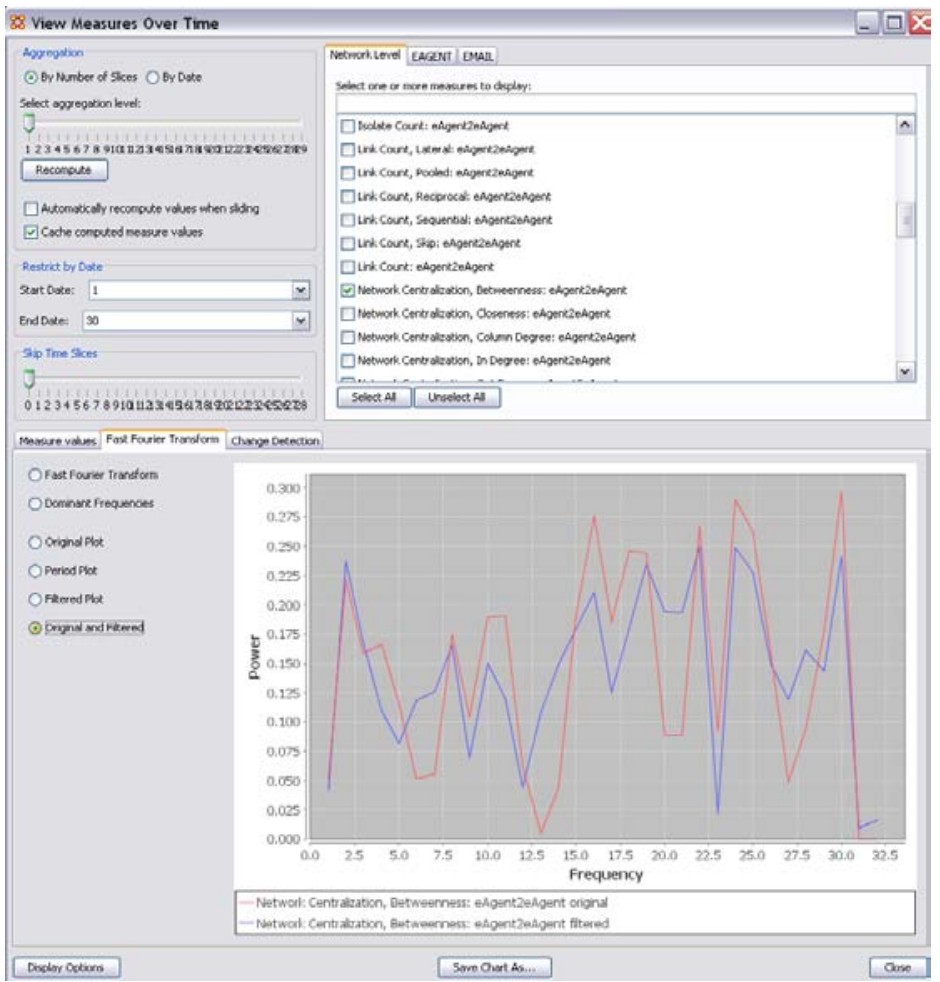




\subsection{STEP 3: Network Change Detection}

The user may also wish to detect statistically significant change in the network over time. The user can select the Change Detection tab shown in the screen capture below. There are three different control chart procedures that can be applied to the signal. We are applying the Cumulative Sum or CUSUM in this example. The user must enter the number of "In-control" networks. This is the number of networks that you must assume to be typical. The procedure actually detects networks that are significantly different from the networks that are "in the first however many networks are selected as in-control". For the CUSUM, the analyst can select a standardized magnitude of change to optimize the procedure for. A novice user should just use the default value of 1 . Finally, the user must determine the procedure's sensitivity to false alarms. The user can select a decision interval, or specify the false positive risk, or specify the expected number of observations until a false positive is reached. For this example, we use a false positive risk of 0.01 , which corresponds to a decision interval of 3.5 and 100 expected observations until a false positive.

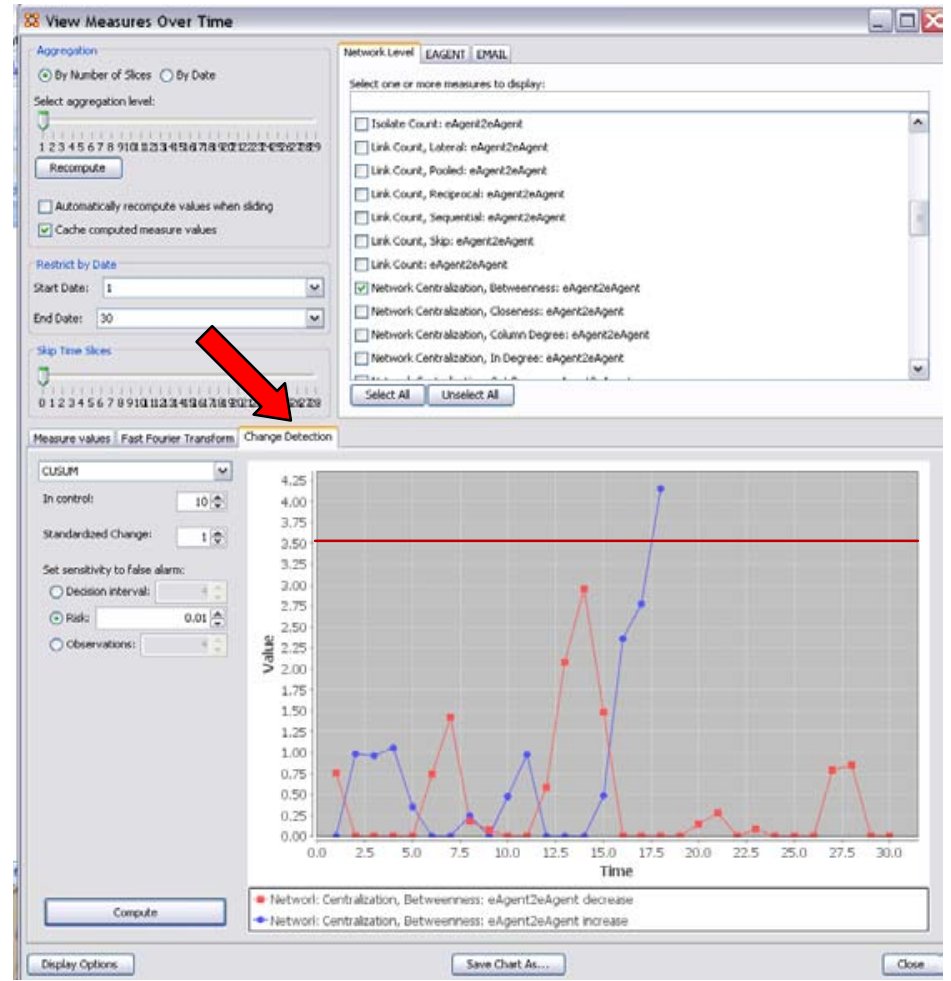

The maroon horizontal line is the decision interval of the change detection procedure. When a plot of the CUSUM crosses the decision interval, the analyst may conclude that a change in network behavior may have occurred. The red line in the plot is the CUSUM statistic for detecting a decrease in the Betweenness Network Centralization. The blue line in the plot is the CUSUM statistic for detecting an increase in the Betweenness Network Centralization. The example above indicates that there may have been a statistically significant change in the network over time. We detect a change in the network on 18 September 2008 (time period 18), when the blue line crosses the 
decision interval. This signals an increase in the Betweenness Network Centralization. The most likely time the change actually occurred was the last time the statistic was 0 , which was 14 September 2008.

The cadet regimental chain of command assumed duties on 18 August. Part of the IkeNet3 experiment was to introduce blackberries to the chain of command and observe the impact. The blackberries were scheduled to be issued to the cadet chain of command on 18 September. They were notified at their weekly meeting on 14 September. All of the cadets were not issued their blackberries until 22 September, but the first blackberry was issued on 18 September. Therefore, dynamic network change detection is successful at detecting this significant event in the organizational behavior of one of three subpopulations monitored. In other words, the behavior only changed for 24 out of 68 individuals in the network. This demonstrates the ability of dynamic network change detection to detect small persistent change in network behavior in a background of noise.

The Over Time Viewer can be used to investigate multiple different network measures, different risks for false positives, and different aggregation levels. The analyst must simply make their selections and then hit the "Compute" button in the Change Detection tab. Different aggregation levels can also be investigated by selecting the aggregation level in the upper right of the interface, hitting "Recompute", then hitting the "Compute" button in the Change Detection tab.

\section{Future Work}

Additional features in the Over Time Viewer will include additional change detection procedures. Currently, the software tool contains three procedures, the Shewhart (1927) x-bar chart, the CUSUM (Page, 1951), and the Exponentially Weighted Moving Average (Roberts, 1959). While these have been demonstrated to be an effective approach for network change detection (McCulloh, 2009) other approaches exist.

There are many factors that contribute to an analyst's choice of change detection procedure. Additional work is still required on the performance of network change detection to determine which of these factors are appropriate for network applications of statistical process control. The Over Time Viewer will continue to incorporate additional change detection procedures to allow users to investigate change detection using the CASOS software suite. An early example is the incorporation of agent level measures for change detection. Although unproven, these features are already present in the Over Time Viewer.

Change detection in longitudinal networks is a relatively new field of active and ongoing research. No doubt, new approaches and concerns will be raised as scientists explore network change. As new methods are developed, we intend to incorporate them in future versions of the Over Time Viewer. 


\section{References}

Carley, K.M. (1991). A theory of group stability. American Sociology Review, 56(3):331-354.

Carley, K.M. (1995). Communication Technologies and Their Effect on Cultural Homogeneity, Consensus, and the Diffusion of New Ideas. Sociological Perspectives, 38(4): 547-571.

Carley, K.M., Diesner, J., Reminga, J., Tsvetovat, M. (2004). Interoperability of Dynamic Network Analysis Software.

Carley, K. M. (2007). ORA: Organizational Risk Analyzer v.1.7.8. [Network Analysis Software]. Pittsburgh: Carnegie Mellon University.

Carrington, P.J., Scott, J., and Wasserman, S. (2007). Models and Methods in Social Network Analysis. Cambridge University Press.

Doreian, P. (1983). On the evolution of group and network structures. II. Structures within structure. Social Networks, 8, 33-64.

Doreian, P., and Stokman, F.N. (Eds.) (1997) Evolution of Social Networks. Amsterdam: Gordon and Breach.

Feld, S. (1997). Structural embeddedness and stability of interpersonal relations. Social Networks, 19, 91-95.

Frank, O. (1991). Statistical analysis of change in networks, Statistica Neerlandica 45 (1991), 283-293.

Holland, P. and Leinhardt, S. (1977). A dynamic model for social networks. Journal of Mathematical Sociology, 5, 5-20.

Holland, P.W., Leinhardt, S. (1981). An exponential family of probability distributions for directed graphs (with discussion). Journal of the American Statistical Association 76, 33-65.

Huisman, M., and Snijders, T.A.B. (2003). Statistical analysis of longitudinal network data with changing composition. Sociological Methods and Research, 32, 253287.

Johnson, J.C., Boster, J.S., and Palinkas, L.A. (2003). Social roles and the evolution of networks in extreme and isolated environments. Journal of Mathematical Sociology, 27, 89-121.

Katz, L. and Proctor, C.H. (1959). The configuration of interpersonal relations in a group as a time-dependent stochastic process. Psychometrika, 24, 317-327.

Krackhardt, D. (1998) Simmelian ties: Super strong and sticky. In Power and Influence in Organizations (eds R. Kramer, M. Neale), pp, 21-38. Sage, Thousand Oaks, CA.

Leenders, R. (1995) Models for network dynamics: a Markovian framework. Journal of Mathematical Sociology, 20, 1-21.

McCulloh, I. (2009). Detecting Changes in a Dynamic Social Network. Ph.D. Thesis, Carnegie Mellon University, Pittsburgh, PA.

McCulloh, I. \& Carley, K. M . (2008). Social Network Change Detection. Carnegie Mellon University, School of Computer Science, Institute for Software Research, Technical Report CMU-ISR-08-116

McCulloh, I., Garcia, G., Tardieu, K., MacGibon, J., Dye, H., Moores, K., Graham, J. M., \& Horn, D. B. (2007a). IkeNet: Social network analysis of e-mail traffic in the Eisenhower Leadership Development Program. (Technical Report, No. 1218). 
Arlington, VA: U.S. Army Research Institute for the Behavioral and Social Sciences.

McCulloh, I., Lospinoso, J., and Carley, K.M. (2007b). Social Network Probability Mechanics. Proceedings of the World Scientific Engineering Academy and Society $12^{\text {th }}$ International Conference on Applied Mathematics, Cairo, Egypt, 2931 December, 2007.

McCulloh, I., Ring, B., Frantz, T.L., and Carley, K.M. (2008). Unobtrusive Social Network Data from Email. In Proceedings of the $26^{\text {th }}$ Army Science Conference. Orlando, FL: U.S. Army.

Newcomb, T.N. (1961). The Acquaintance Process. Holt, Rinehart and Winston, New York .

Page, E.S. (1961). Cumulative Sum Control Charts. Technometrics 3, 1-9.

Roberts, S.V. (1959) Control chart tests based on geometric moving averages. Technometrics 1, 239-250.

Robins, G. and Pattison, P. (2007) Interdependencies and Social Processes: Dependence Graphs and Generalized Dependence Structures. In: P. Carrington, J. Scott and S. Wasserman, Editors, Models and Methods in Social Network Analysis, Cambridge University Press, New York, 192-214.

Robins, G. and Pattison, P. (2001) Random graph models for temporal processes in social networks. Journal of Mathematical Sociology, 25, 5-41.

Sampson, S.F., (1969). Crisis in a cloister. Ph.D. Thesis. Cornell University, Ithaca.

Sanil, A., Banks, D., and Carley, K.M. (1995). Models for evolving fixed node networks: Model fitting and model testing. Social Networks, 17, 1995.

Shewhart, W.A. (1927). Quality Control. Bell Systems Technical Journal.

Snijders, T.A.B. (2007). Models for longitudinal network data. In: P. Carrington, J. Scott and S. Wasserman, Editors, Models and Methods in Social Network Analysis, Cambridge University Press, New York, 148-161.

Snijders, T.A.B. (1990) Testing for change in a digraph at two time points. Social Networks, 12, 539-573.

Snijders, T. A. B., Van Duijn, M.A.J.. (1997). Simulation for Statistical Inference in Dynamic Network Models. In Simulating Social Phenomena, (Ed. R. Conte, R. Hegselmann, and P. Tera) Berlin: Springer, pp. 493-512.

Snijders, T.A.B. (2001) The statistical evaluation of social network dynamics. In: Sobel, M.E. and Becker, M.P. (Eds) Sociological Methodology, 361-395. Boston: Basil Blackwell.

Wasserman, S. (1980). Analyzing social networks as stochastic processes. Journal of American Statistical Association, 75, 280-294.

Wasserman, S. (1977). Stochastic Models for Directed Graphs. Ph.D. dissertation, Harvard University, Department of Statistics, Cambridge, MA.

Wasserman, S. (1979). A stochastic model for directed graphs with transition rates determined by reciprocity. In Sociological Methodology 1980 (Ed. Schuessler, K.F.) San Francisco: Jossey-Bass, 392-412.

Wasserman, S., \& Faust, K. (1994). Social Network Analysis: Methods and Applications. New York: Cambridge University Press.

Wasserman, S., Iacobucci, D. (1988). Sequential Social Network Data. Psychometrika 53:261-82. 
Van de Bunt, G.G., Van Duijin, M.A.J., and Snijders, T.A.B. (1999). Friendship networks through time: An actor-oriented statistical network model. Computational and Mathematical Organization Theory, 5, 167-192. 